livraisons

d'Histoire

de l'Architecture

\section{Livraisons de l'histoire de l'architecture}

26 | 2013

Les ministres et les arts

\title{
Finances et arts pendant la Révolution et le Premier Empire. L'exemple du Garde-Meuble
}

Finances and arts during the Revolution and Empire: the example of the Gardemeuble

Kunst und Finanzen während der Revolution und des Ersten Kaiserreiches. Das Beispiel der Mobiliarverwaltung des Garde-Meuble

\section{Aleth Tisseau des Escotais}

\section{OpenEdition}

\section{Journals}

Édition électronique

URL : http://journals.openedition.org/lha/341

DOI : 10.4000/lha.341

ISSN : 1960-5994

Éditeur

Association Livraisons d'histoire de l'architecture - LHA

Édition imprimée

Date de publication : 10 décembre 2013

Pagination : 123-137

ISSN : 1627-4970

Référence électronique

Aleth Tisseau des Escotais, « Finances et arts pendant la Révolution et le Premier Empire. L'exemple du Garde-Meuble », Livraisons de l'histoire de l'architecture [En ligne], 26 | 2013, mis en ligne le 10 décembre 2015, consulté le 30 avril 2019. URL : http://journals.openedition.org//ha/341 ; DOI : 10.4000/lha.341 


\section{FINANCES ET ARTS PENDANT LA RÉVOLUTION ET LE PREMIER EMPIRE. L'EXEMPLE DU GARDE-MEUBLE}

Sous l'Ancien Régime, le roi et son entourage ont pris l'habitude de jouer leur rôle de mécène dans le domaine des arts décoratifs par le biais du Garde-Meuble. Le directeur du Garde-Meuble lui-même, la plupart du temps amateur éclairé, sait repérer les artistes de talent et les employer au service du roi et de ses propres ambitions. Entre 1774 et 1784 par exemple, ce sont plus de sept cents meubles que Riesener, alors seul ébéniste de la Couronne, livre au Garde-Meuble ${ }^{1}$. Il bénéficie pour cela de l'appui du directeur Pierre-Élisabeth de Fontanieu, mais aussi de la reine Marie-Antoinette, des comtesses de Provence et d'Artois et de Mesdames. Ces commandes ont un coût. Les finances royales, et par conséquent nationales, sont lourdement mises à contribution.

La logique change du tout au tout avec la rupture de la Révolution. Si le premier inspecteur, Jean-Bernard Restout (20 août 1792-22 avril 1793) peut être considéré comme un connaisseur en sa qualité de peintre et graveur, c'est loin d'être le cas de ses successeurs, François-Louis Bayard, Louis-François Dubois et Nicolas-Auguste Villette pour la période révolutionnaire, Étienne-Jacques Calmelet puis Alexandre-Jean Desmazis pour la période impériale. Les représentants de l'exécutif ne sont pas plus passionnés d'arts. La sobriété républicaine commence à peine à être remise en cause sous le Directoire que̊ le coup d'État du 18 brumaire ouvre la voie au général Bonaparte, dont les rapports aux arts, tout comme à la religion, sont avant tout pragmatiques.

Privé d'une personnalité éclairée qui impose une ligne directrice aux domaines de l'ameublement et du textile, le Garde-Meuble se retrouve coupé de ses financements traditionnels. Il est désormais soumis à l'état fluctuant des finances, qui sont devenues une priorité nationale. L'institution connaît dès lors une rigueur budgétaire sans précédent pendant les années révolutionnaires, puis un renouveau calculé sous l'Empire.

\section{Les années révolutionnaires : gestion rigoureuse, économies et productivité}

Dans le contexte économique et financier critique que constituent les années révolutionnaires, la gestion des crédits fait l'objet d'un soin particulier, dans le but de réduire les dépenses, et parfois de créer des bénéfices.

1. Voir Stéphane Castelluccio, Le Garde-Meuble de la couronne et ses intendants du XVI siècle au XVIII siecle, Paris, CTHS, 2004, 333 p., p. 184. 


\section{Des dépenses contrôlées et réduites}

Au niveau de l'organisation des comptes financiers, la distinction entre dépenses fixes et dépenses extraordinaires, générale sous l'Ancien Régime, demeure. Les dépenses fixes, qui sont des dépenses régulières, autorisées au départ par le ministre de l'intérieur, puis reconduites d'un état à l'autre, peuvent faire l'objet de paiements mensuels ou par quartier. Ce sont les états par quartier ou trimestre qui dominent pendant la période révolutionnaire. Les dépenses extraordinaires sont quant à elles des dépenses ponctuelles, qui font l'objet d'une autorisation spéciale et d'un mémoire qui leur est propre. Ces deux types de dépenses sont réglés directement par la Trésorerie nationale ou la comptabilité du ministre de l'intérieur, sans passer par le Garde-Meuble, qui se contente d'en apporter les éléments.

Pendant la période révolutionnaire, on ajoute une troisième catégorie de dépenses, les dépenses urgentes. Ces dépenses comprennent tous les menus frais que le GardeMeuble règle sur les fonds d'avance qui lui sont versés. Un compte rendu doit en être réalisé chaque décade. Une lettre du 27 fructidor an II (13 septembre 1794) ${ }^{2}$ fixe à 3000 livres les fonds d'avance. La possibilité de disposer de ce fonds d'avance est le résultat de réclamations répétées de l'inspecteur, rejetées dans un premier temps par le ministre de l'intérieur Garat ${ }^{3}$, avant d'être approuvées par son successeur Paré.

Quel que soit le type de dépenses dont il est question, un aspect majeur est souligné en permanence par le ministre de l'intérieur et la commission des Revenus nationaux: il s'agit de la nécessité de rendre des comptes. Garat par exemple s'exprime ainsi dans une lettre du 10 juin $1793^{4}$ adressée à l'inspecteur provisoire Bayard : "Les états et les rapports doivent être considérés par vous comme un objet essentiel et insuppléable de votre activité, parce qu'ils écartent l'arbitraire, précisent les objets et sont dès lors indispensables à votre responsabilité comme à la mienne ". Rendre des comptes est un devoir pour tout agent public selon la Déclaration des droits de l'homme et du citoyen.

Les services du ministre de l'intérieur et de la commission des Revenus nationaux examinent chaque compte des dépenses extraordinaires et urgentes transmis par l'inspecteur. Ils émettent des observations sur chaque frais mentionné dans le compte, et concluent en déclarant l'article soit " passé " ou " bon ", soit " à rejeter ". Dans un de ces examens de compte ${ }^{5}$, la commission des Revenus nationaux explique à Bayard le processus à suivre pour que la gestion des dépenses extraordinaires et urgentes soit réalisée correctement. Après avoir été visées par l'inspecteur, ces dépenses devraient passer par le vérificateur Sulleau avant de pouvoir être payées

2. Voir Arch. nat., $\mathrm{O}^{2} 374$.

3. Voir Arch. nat., $\mathrm{O}^{2} 374$, la lettre adressée par le ministre de l'intérieur à l'inspecteur, datée du 10 juin 1793.

4. Voir Arch. nat., $\mathrm{O}^{2} 374$.

5. Voir Arch. nat., $\mathrm{O}^{2} 476$, le compte des dépenses extraordinaires et urgentes faites avec les 3000 livres mises à la disposition du Garde-Meuble par l'ordonnance de la Commission en date du 14 thermidor an III ( $1^{\text {er }}$ août 1795$)$. 
par Hamelin, dépositaire des fonds du Garde-Meuble. On constate que les fonctions d'ordonnateur, de comptable et de contrôleur sont clairement définies et distinguées.

Ce sérieux budgétaire n'est pas suffisant. S'y ajoute une rigueur budgétaire appuyée. Faire faire des économies à l'État apparaît alors comme une marque importante de zèle patriotique, comme une sorte de profession de foi républicaine. Les économies se réalisent en permanence, dans la gestion de l'institution et même, dans l'ameublement des bâtiments publics, au moins jusqu'au Directoire.

Dans la gestion de l'institution, le premier facteur d'économie est la réduction du personnel. Le personnel coûte cher, et il devient de plus inutile au fur et à mesure de la liquidation du Garde-Meuble. Quand des besoins ponctuels de personnel se font néanmoins sentir, l'inspecteur n'hésite pas à recourir alors à des journaliers, qui lui reviennent moins cher, notamment parce qu'ils ne bénéficient pas d'avantages tels que le logement. Dubois a bien compris ce mécanisme dès son entrée en fonction : "Citoyen Ministre, lorsque, m'accordant ta confiance, tu me chargeas de l'inspection générale du Garde-Meuble et des Menus réunis, j'ai dû pour la mériter et par devoir entrer dans les vues d'économie dont tu es animé pour la chose publique et t'en soumettre les résultats. Mon premier soin a été de prendre connaissance de l'état des employés dans cette administration, de l'occupation et de l'utilité de chacun d'eux ", écrit-il le 16 nivôse an II (5 janvier 1794) ${ }^{6}$, un mois après sa nomination.

Le second facteur d'économie dans la gestion du Garde-Meuble consiste à réduire tous les menus frais de fonctionnement. C'est ainsi que l'inspecteur propose par exemple, dans une lettre du 20 février 1793 adressée au ministre de l'intérieur ${ }^{7}$, d'utiliser pour le transport des meubles les chevaux du ministère de la guerre lorsqu'ils ne sont pas employés par celui-ci. La question des chevaux et des voitures est un problème crucial, la République étant d'une façon générale à court de chevaux $^{8}$. Chaque action du Garde-Meuble doit être revue dans un objectif d'économie. Le fait de devoir justifier de façon particulièrement précise chaque dépense, dans un état particulier s'il s'agit d'une dépense courante, ou sur l'état des dépenses extraordinaires et urgentes dans les autres cas, en sachant que ces états seront relus avec attention, limite les abus, qu'il s'agisse de faux frais ou de frais dont le coût serait surestimé.

La gestion de Bayard est jugée globalement positive par la commission des Revenus nationaux : "Nous ne pouvons qu'applaudir au zèle et à l'empressement que tu mets à la recherche des moyens de rendre et plus active et moins dispendieuse à la République l'administration qui t'est confiée?." Il faut noter que Bayard a une formation de négociant. Le fait qu'un tel personnage ait été choisi à

6. Voir Arch. nat., $\mathrm{O}^{2} 417$.

7. Voir Arch. nat., $\mathrm{O}^{2} 375$.

8. Voir Arch. nat., $\mathrm{O}^{2} 374$, la lettre adressée par Bochet à Bayard, datée du $3^{\mathrm{e}}$ sans-culottide an II (19 septembre 1794). "Les chevaux sont très rares ", note Bochet.

9. Voir Arch. nat., $\mathrm{O}^{2} 374$, la lettre datée du $3^{\text {e }}$ sans-culottide an II (19 septembre 1794). 
la tête du Garde-Meuble est extrêmement révélateur : la gestion prime désormais sur la mission artistique qu'incarnait encore son prédécesseur, le peintre et graveur Jean-Bernard Restout.

Faire des économies dans la gestion du Garde-Meuble s'avère être une chose facile, ou du moins réalisable. Il n'en va pas de même dans le domaine de l'ameublement des établissements publics. En effet, le Garde-Meuble doit obéir aux souhaits des divers ministres et commissions, une fois l'approbation du ministre de l'intérieur obtenue, et sa marche de manœuvre est relativement faible. Il peut agir sur le processus de fabrication des meubles et textiles, en le surveillant de plus près ou en recourant à des meubles réquisitionnés, mais à l'arrivée le produit doit être tel qu'il a été exigé qu'il soit. L'inspecteur ne peut se permettre de discuter une demande. Sous la Convention, l'austérité domine, mais le faste et les goûts de luxe font leur réapparition sous le Directoire.

\section{Ventes, destructions et loteries}

Les choses vont plus loin lorsqu'on demande au Garde-Meuble, non seulement de faire des économies sur son fonctionnement général, mais également de produire des bénéfices. Il doit se trouver des sources de revenus et contribuer à l'effort financier national. Pour cela, il organise des ventes, des destructions, et contribue au dispositif des loteries publiques.

Des ventes de mobilier du Garde-Meuble ont déjà eu lieu à plusieurs reprises sous l'Ancien Régime. En 1645, un arrêt du 10 juin ordonne de dresser l'inventaire des meubles des châteaux du Louvre et du Petit-Bourbon et de vendre aux enchères " ceux qui seront inutiles ou inutilisable ${ }^{10}$ ". En $1736,1741,1751$ et 1752 , sous Gaspard-Moïse-Augustin de Fontanieu, des ventes sont à nouveau organisées. Thierry de Ville-d'Avray en met en place lui aussi, au cours des années 1785 et 1786. La plupart du temps, ces ventes ne concernent que des objets de peu de valeur. Les opérations des années 1751 et 1752 font exception, et provoquent des pertes non négligeables pour les collections du Garde-Meuble.

Pendant la Révolution, ces ventes prennent une ampleur bien supérieure. Le décret du 22 octobre 1792 ordonne la vente du mobilier des maisons royales. Un décret d'application du 10 juin $1793^{11}$ marque le véritable point de départ de ce mouvement. Les commissaires de la Convention sont élus quinze jours plus tard, afin d'encadrer le processus qui débute le 20 août à Rambouillet, le 25 août à Versailles, le 6 octobre à Marly, puis s'étend à partir du printemps 1794 à Saint-

10. Voir Michel Le Pensant, Arrêts du conseil du roi. Règne de Louis XIV (20 mai 1643-8 mars 1661), t. I, Paris, Imprimerie nationale, 1976, p. 38, n 371, cité dans Le Garde-Meuble de la Couronne et ses intendants $d u X V I^{e}$ au XVIII siecle, op. cit., p. 37.

11. Voir Rémi Gaillard, "Après qu'il s'est assemblé marchands fripiers, tapissiers, revendeurs et autres citoyens ". Étude institutionnelle des ventes révolutionnaires du mobilier royal. Versailles, Marly, SaintCloud, Bellevue (1793-1795), thèse sous la direction de Jean-Michel Leniaud, École nationale des chartes, s.l., s.n., 2011, 2 vol., vol. I. 
Cloud et du printemps 1795 à Bellevue. Aux ventes de mobilier des maisons royales s'ajoutent les ventes de mobilier du Garde-Meuble de Paris.

Ces opérations ont un but politique et économique évident, mais il ne faut pas oublier qu'elles ne sont pas toujours le résultat d'une volonté délibérée. Devant l'insécurité qui règne et les vols qui sont perpétrés, la vente apparaît parfois comme un moindre mal: puisque de toute façon les meubles ne peuvent être conservés, autant les vendre, ils auront ainsi une certaine utilité. C'est la réflexion que fait le ministre de l'intérieur Garat, le 19 mai 1793 , dans une lettre adressée à Bayard ${ }^{12}$. Après que deux vols ont été commis à Trianon, la vente du mobilier du palais est envisagée comme une des solutions au problème. La vente est préférable au vol.

Le rôle du Garde-Meuble dans ces aliénations est décisif, même s'il agit en permanence sous le contrôle du pouvoir. Dans le cas des résidences royales, il lui revient de dresser l'état des meubles à sacrifier ${ }^{13}$; il est donc largement responsable de la nature et de la quantité des objets dont on se sépare. Dans le cas des ventes organisées au Garde-Meuble de la place de la Révolution, il se charge bien évidemment de réaliser l'inventaire des meubles dont on se défait, mais il a en plus de cela la responsabilité de l'organisation des ventes, qui consiste essentiellement à s'assurer de la présence de représentants de l'État ${ }^{14}$. Car aucune vente ne peut avoir lieu sans la surveillance de deux membres de la Convention puis, à partir de novembre 1793, de deux commissaires du Conseil exécutif provisoire. Ces représentants du peuple, intéressés au produit des enchères, ont une fonction capitale dans le processus de vente.

Un autre rôle que s'attribue le Garde-Meuble dans l'organisation de l'aliénation du mobilier national consiste à s'assurer de la rentabilité des ventes. La multiplication des ventes particulières à Paris rend les opérations d'aliénation moins avantageuses. Cependant, il reste préférable de vendre à Paris qu'ailleurs, en raison du

12. Voir Arch. nat., $\mathrm{O}^{2} 374$. Le ministre s'exprime en ces termes : "Un rapport qui vient de m'être fait officiellement d'un second vol commis récemment sur les plombs des combles de Trianon me détermine, Citoyen, à prendre les mesures les plus propres à mettre à l'abri de pareilles récidives, ainsi que des dégradations et vols qui pourraient en être la suite, le mobilier renfermé dans ce cidevant château. Vous voudrez bien, en conséquence, me faire un très prompt rapport sur la possibilité de mettre en vente une partie de ce mobilier. "

13. Voir Arch. nat., $\mathrm{O}^{2} 374$, l'extrait du registre des délibérations du comité d'aliénation daté du

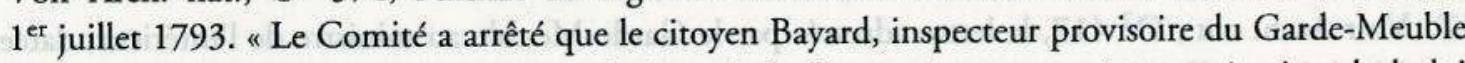
de la République, remettrait aux commissaires de la Convention, nommés en exécution de la loi du 10 juin, un état des meubles placés dans les différentes maisons de la ci-devant Liste civile qu'il lui paraît plus pressant et plus intéressant de faire vendre."

14. Voir Arch. nat., $\mathrm{O}^{2} 417$, la lettre adressée par Dubois au ministre de l'intérieur, datée du 18 frimaire an II (8 décembre 1793) : "Citoyen, tu voudras bien te rappeler que je t’ai prévenu que la vente du mobilier, Maison du Garde-Meuble national, à Paris, reprendrait son cours le primidi de la première décade de nivôse. En conséquence, la nomination et la présence des deux commissaires du pouvoir exécutif étant indispensable, tu voudras bien y pourvoir, et me donner tes ordres. " Les deux commissaires du conseil exécutif pour ces ventes de nivôse (décembre 1793-janvier 1794) sont les citoyens Godefroy et Poisson, voir Arch. nat., $\mathrm{O}^{2}$ 417, la lettre adressée par Dubois au ministre de l'intérieur, datée du 25 nivôse an II (14 janvier 1794). 
nombre de marchands qui y résident. C'est pourquoi l'inspecteur demande, entre autres, que les meubles de la maison de Madame Élisabeth à Montreuil et du Garde-Meuble de Monsieur à Versailles ne soient pas vendus à Versailles ${ }^{15}$, et que les objets " d'un haut prix, dont on pourrait tirer plus d'avantage à Paris ${ }^{16}$ " du cidevant marquis de Polignac ne demeurent pas dans le Loir-et-Cher. Il faut éviter également de tenir deux ventes d'importance en même temps à Paris ${ }^{17}$, car cela réduit le nombre de marchands présents à chacune des ventes, et la hausse des enchères s'en trouve limitée. Le Garde-Meuble essaie de rationaliser les ventes, afin d'en tirer le maximum de profit.

Une variante de la vente consiste dans la délivrance de meubles aux fournisseurs de la République, en paiement de leurs fournitures. Les "capitaines grecs" par exemple, des négociants qui profitent de l'état critique du commerce en France, reçoivent au cours de l'an IV et de l'an V (1795-1797) des tapisseries, des étoffes, des tableaux et des estampes en nombre conséquent ${ }^{18}$. De même, le 24 pluviôse an IV (13 février 1796) ${ }^{19}$, on décide de céder quatre pièces de tapisseries de Beauvais représentant les quatre parties du monde, emblématiques de la Révolution américaine, au citoyen Sadler, négociant des États-Unis d'Amérique, qui en a fait la demande. Ayant peu de hauteur et étant tachées de signes de féodalité difficiles à faire disparaître, elles sont peu convenables à l'ameublement du GrandLuxembourg et leur cession n'est pas une vraie perte pour la nation. Finalement, Sadler renonce à cette acquisition, et ces tapisseries sont remises au fournisseur Abraham Alcan ${ }^{20}$. Le paiement des fournisseurs, tout comme la remise d'objets en déduction de créances sur la République, entame sérieusement les collections du Garde-Meuble.

Toujours dans un objectif de profit, le Garde-Meuble organise des fontes et destructions diverses, comme cela se pratiquait déjà sous l'Ancien Régime. À l'hiver 1689-1690, Louis XIV fait fondre son mobilier d'argent pour financer sa politique de guerres. Des fontes d'objets d'or et d'argent ont lieu en 1726 et en 1751 sous Louis XV, tandis que le Garde-Meuble fait brûler en mars 1746 et en novembre

15. Voir Arch. nat., $\mathrm{O}^{2} 375$, la lettre adressée par le Garde-Meuble au ministre de l'intérieur, datée du 20 février 1793.

16. Voir Arch. nat., $\mathrm{O}^{2} 377$, la lettre adressée par Bayard aux administrateurs du département de Loiret-Cher, datée du 23 mai 1793.

17. Voir Arch. nat., $\mathrm{O}^{2} 417$, la lettre adressée par le Garde-Meuble au ministre de l'intérieur, datée du 29 nivôse an II (18 janvier 1794), dans laquelle est évoquée la concurrence potentielle que la vente de l'hôtel de Coigny ferait aux ventes du Garde-Meuble national. Il ne faudrait vendre à la maison Coigny que les décades où l'on ne vend pas au Garde-Meuble, ou faire en sorte que la Commission du Garde-Meuble, qui est "infiniment économique ", se charge de tout. On craint sinon de devoir suspendre à nouveau les ventes, comme cela s'était produit en brumaire (octobre-novembre 1793).

18. Voir Arch. nat., $\mathrm{O}^{2} 464$, notamment l'état des tableaux et estampes demandés par les capitaines grecs.

19. Voir Arch. nat., $\mathrm{O}^{2} 385$, cité dans Jules-Joseph Guiffrey, Destruction des plus belles tentures du mobilier de la Couronne en 1797, Nogent-le-Rotrou, imprimerie de Daupeley-Gouverneur, 1887, 34 p., p. 31 .

20. Voir Arch. nat., $\mathrm{O}^{2} 464$. 
1759 des meubles de brocart pour en retirer le métal précieux ${ }^{21}$. Sous le règne de Louis XVI enfin, Thierry brûle des étoffes au cours des années 1784-1785, fait retailler les diamants de façon catastrophique, et envoie une partie de l'orfevrerie royale à la Monnaie pendant l'automne 1789, pour participer à ces dons patriotiques qui ont pour but de pallier l'effondrement des recettes fiscales.

Il n'est donc pas étonnant que le Garde-Meuble national ait recours à des fontes et à des destructions pour remplir ses caisses, il agit dans la lignée de pratiques du Garde-Meuble de la Couronne. L'opération la plus ample qui a lieu est la destruction d'anciennes tapisseries. En tout, dix-huit tentures, comprenant cent quatre-vingt dix tapisseries, sont détruites en quelques minutes à la Monnaie, au cours de l'année 1797, pour une somme de 65 ou 66000 francs $^{22}$. Les opérations concernant les tapisseries sont les plus spectaculaires, mais elles sont loin d'être isolées. La loi du 10 juin 1793 prévoit l'envoi à la Monnaie des objets d'orfevrerie qui n'ont pas de plus-value liée à un caractère d'œuvre d'art ${ }^{23}$. On peut citer dans cet ordre d'idées les dorures du mobilier de la Liste civile qui sont conduites à la Monnaie nationale ${ }^{24}$; ou, en ce qui concerne les objets de moindre valeur, les étoffes avec motifs de fleurs de lys qui sont transformées en papier ${ }^{25}$, les vieux lits qui deviennent du bois de chauffage ${ }^{26}$. Globalement, toute la période est parcourue d'envois d'objets précieux à la Monnaie ${ }^{27}$. Parfois, le profit de la destruction n'est pas matériel. Par exemple, le Garde-Meuble doit fournir des objets rappelant la royauté pour le bûcher de la fête nationale ${ }^{28}$. Le bénéfice est alors moral, les effets brûlés contribuent à l'instruction républicaine du peuple.

Le troisième point qui permet au Garde-Meuble de générer des profits est le phénomène de la loterie. Ce jeu de hasard aux origines très anciennes a été pratiqué officiellement pour la première fois en France en 1539. Il s'y développe, malgré les vifs débats qu'il occasionne, et les sommes produites deviennent tellement importantes que la royauté récupère les loteries à son seul profit dans la seconde moitié du XVIII ${ }^{\mathrm{e}}$ siècle.

21. Voir Le Garde-Meuble de la Couronne et ses intendants du XVI au XVIII siècle, op. cit., p. 154-155.

22. Voir " Destruction des plus belles tentures du mobilier de la Couronne en 1797 ", op. cit., p. 3-6.

23. Voir Jean Baptiste Duvergier, Collection complète des lois, décrets, ordonnances, règlements, avis du Conseil d'État, publiée sur les éditions officielles du Louvre; de l'Imprimerie nationale, par Baudoin; et $d u$ bulletin des lois, de 1788 à 1830 inclusivement, par ordre chronologique, Paris, A. Guyot et Scribe, 1824-1949, 158 vol., t. V, p. 333-337. Article 19: "Les ouvrages d'orfevrerie qui ne sont pas précieux par leur travail, ainsi que les cuivres et bronzes qui ne peuvent être regardés comme monuments d'arts, et qui ne tirent pas de la façon une plus-value considérable, seront, si fait n'a été, portés à la Monnaie pour y être convertis en espèces [...] ".

24. Voir Arch. nat., $\mathrm{O}^{2} 374$, la lettre adressée par la commission des Revenus nationaux à l'inspecteur, datée du 18 fructidor an II (4 septembre 1794).

25. Voir Arch. nat., $\mathrm{O}^{2} 374$, la lettre adressée par la commission des Revenus nationaux à l'inspecteur, datée du 27 fructidor an II (13 septembre 1794).

26. Voir Arch. nat., $\mathrm{O}^{2} 476$.

27. Voir Arch. nat., $\mathrm{O}^{2} 464$.

28. Voir Arch. nat., $\mathrm{O}^{2} 374$, la lettre adressée par le ministre de l'intérieur à l'inspecteur, datée du 8 août 1793. Sont concernés " le trône, des accessoires et tous autres attributs de la royauté qui pourraient exister au Garde-Meuble ». 
Pendant la période révolutionnaire, deux grandes loteries nationales au moins sont tirées. Les loteries font l'objet d'un décret du 27 vendémiaire an IV (19 octobre 1795), qui établit un délai de six mois pendant lequel les porteurs de billets gagnants aux loteries nationales de maisons, meubles et effets peuvent réclamer les lots qui leur reviennent. Au-delà, ils sont déchus de toute prétention sur l'objet de leur lot, qui reste au profit de la République.

Ces loteries concernent le Garde-Meuble, car il pourvoit aux lots en meubles, participant ainsi à l'attractivité du jeu, et aux bénéfices qui en sont générés par conséquent. Établie par le décret du 29 germinal an III (18 avril 1795), et tirée les 2 et 12 fructidor suivants ( 19 et 29 août), la première loterie révolutionnaire ne coûte pas grand chose aux collections du Garde-Meuble, car les porteurs de billets gagnants tardent à se faire connaître. Seul le citoyen Lullin se présente, et remporte avec lui une paire de draps de toile de Hollande d'une valeur de 1600 francs $^{29}$. La seconde loterie est tirée en brumaire an IV (octobre-novembre 1795). Les gagnants se présentent en nombre plus important, et les objets attribués sont de plus grande valeur : deux paires de bras en bronze doré d'un montant de 62000 francs; une table de trictrac en acajou et ébène d'un montant de 3400 francs; une commode en acajou valant 24000 francs; deux vases de porcelaine de Sèvres d'une valeur de 3400 francs; enfin, une table à écrire en acajou d'un montant de 6000 francs. Même si ce phénomène reste limité, il participe à la dilapidation des collections du Garde-Meuble.

Le Garde-Meuble, face aux difficultés économiques de la période révolutionnaire, sait s'adapter et limiter les coûts qu'il occasionne en réduisant ses frais de fonctionnement, en recourant à des meubles réquisitionnés et en générant des profits, principalement par le biais de ventes et de fontes dont l'ampleur dépasse ce qu'avait pu connaître l'Ancien Régime. Il parvient également à participer à la bonne santé financière de la République en contribuant au paiement de ses fournisseurs et créanciers et en soutenant le système des loteries. Cette exigence de productivité imposée au Garde-Meuble est une constante de l'époque révolutionnaire, il semble que les transformations politiques n'aient pas eu d'influence sur cet impératif. Alors que sous l'Ancien Régime les aliénations et les destructions s'accompagnaient de nouvelles commandes qui renouvelaient le mobilier royal, la République ne cherche pas à compenser ces pertes, qui amenuisent les collections du GardeMeuble.

Chassé de son hôtel par le ministère de la marine, privé de ses meubles et de ses ressources financières, le Garde-Meuble est mis en liquidation à partir du $1^{\text {er }}$ messidor an V (19 juin 1797). Dans des locaux réduits, occupés par un personnel ne dépassant pas quinze agents, avec la responsabilité d'un mobilier qui a fondu sous l'effet des ventes et de l'abondance des demandes des établissements publics, l'ancien Garde-Meuble accomplit des missions désormais limitées. Sa disparition totale intervient le 30 floréal an VI (19 mai 1798).

29. Voir Arch. nat., $\mathrm{O}^{2} 425$, l'état sommaire de la composition actuelle du Garde-Meuble, des opérations et du montant des dépenses faites pendant la gestion du citoyen Villette, directeur général de cet établissement, du 22 frimaire au 30 floréal dernier, daté du 16 messidor an IV (4 juillet 1796). 


\section{Les années impériales: un renouveau calculé}

La fin de la mutualisation que créait le Garde-Meuble national oblige chaque établissement public à gérer lui-même son mobilier et, si sa taille l'impose, à édifier en son sein un service qui remplisse ces fonctions. L'héritier le plus direct du Garde-Meuble de la nation est dans un premier temps le service de l'ameublement du Directoire, au Luxembourg. Le départ des Consuls aux Tuileries provoque son déclin et la formation d'un Garde-Meuble du Premier Consul pour les palais des Tuileries et de Saint-Cloud, petite organisation dont ni le personnel ni le budget n'ont quoi que ce soit de commun avec les Garde-Meubles du passé - même le service de la liquidation du Garde-Meuble employait un personnel plus nombreux - ou de l'avenir. Le Garde-Meuble de la Maison du Premier Consul est sans doute largement suffisant pour un militaire habitué à la vie en campagne. Mais il ne peut suffire à un Empereur qui veut rivaliser avec les souverains du passé et ses homologues européens. Il lui faut une ampleur toute différente, du personnel et des crédits en quantité.

L'article 15 du sénatus-consulte du 28 floréal an XII (18 mai 1804), qui proclame Bonaparte "empereur des Français ", attribue à Napoléon la même Liste civile que celle qui avait été accordée à Louis XVI en 1791, dont la journée du 10 août 1792 était venue sonner le glas. Le 28 messidor an XII (17 juillet 1804), Napoléon approuve le règlement fixant l'organisation de sa Maison, qui possède le Mobilier impérial dans ses attributions. Le 13 brumaire an XIII (4 novembre 1804) ${ }^{30}$, Étienne Jacques Calmelet, ancien homme d'affaires de l'impératrice Joséphine, est nommé administrateur du Mobilier impérial.

\section{L'apparition d'un budget et les différentes étapes de sa constitution}

Sous la Révolution, les troubles économiques et financiers et le précepte de sobriété républicaine ont condamné le Garde-Meuble à une parcimonie budgétaire contradictoire avec la logique de faste qui avait été la sienne. Ce précepte d'économie a eu un mérite, celui d'inculquer à l'institution des prémices de rigueur dans sa gestion, que la période impériale va démultiplier et adapter à des budgets plus conséquents.

On distingue alors quatre secteurs de dépenses, répartis entre dépenses ordinaires et dépenses extraordinaires. Le premier concerne le personnel, le second les gratifications et l'habillement, et le troisième l'entretien des palais impériaux : ils constituent les dépenses ordinaires. Le quatrième secteur regroupe l'ameublement et les nouveaux achats, et constitue les dépenses extraordinaires. On remarque que le système binaire continue d'être employé comme par réflexe, mais qu'il ne correspond plus à l'organisation effective des dépenses.

Cette nouvelle gestion des dépenses du Garde-Meuble est révélatrice d'un phénomène plus profond et plus révolutionnaire : l'apparition d'une réflexion à long

30. Voir Arch. nat., $\mathrm{O}^{2} 781$, dossier 3. 
terme sur les finances, qui sont désormais envisagées plusieurs mois à l'avance, conçues sur une durée d'un an, et rectifiées en cours d'année si le besoin s'en fait sentir. On peut dorénavant parler véritablement de l'existence d'un budget. Chaque mois de septembre voit se tenir des réunions de préparation du budget de l'année suivante. L'empereur se fait présenter à cette occasion le projet de budget du Garde-Meuble par l'intendant général. Cela ne signifie pas pour autant que l'intendant serait à l'origine de ce projet. C'est l'administrateur qui veille à sa mise en forme et l'envoie ensuite à l'intendant avant la date prescrite ${ }^{31}$. Nous conservons plusieurs projets de budget du Mobilier impérial. Ces projets sont fondés sur la mise en parallèle du budget de l'année précédente et des prévisions pour l'année à venir. Dans leur structure, ils reprennent les grandes lignes des budgets définitifs, avec la division théorique entre dépenses ordinaires et dépenses extraordinaires, accompagnée d'une subdivision plus pragmatique des dépenses ordinaires en plusieurs chapitres.

Durant la décennie révolutionnaire, le contrôle des dépenses au sein du GardeMeuble était une priorité pour l'institution. Mais les modalités de ce contrôle n'étaient pas encore systématiques. La séparation entre ordonnateur, comptable et vérificateur perdure sous l'Empire. Cependant, la première étape, celle qui voit la dépense être ordonnée, se subdivise en plusieurs moments. Pendant la Révolution, l'inspecteur ou directeur devait faire accepter les opérations par le ministre de l'intérieur ou par la commission des Revenus nationaux seulement. Sous l'Empire, les demandes des concierges sont présentées au grand maréchal du Palais, avant de parvenir à l'administrateur, qui se charge à son tour de les transmettre à l'intendant général, qui les signale éventuellement à l'empereur ${ }^{32}$. Le nombre de décideurs est démultiplié, et leurs exigences sont strictes en ce qui concerne la qualité des mémoires qu'on leur présente. Galle, par exemple, se voit refuser la signature d'un mémoire en 1810 par l'intendant général ${ }^{33}$. Les responsables sont nombreux et difficiles à contenter.

La deuxième étape est celle de la vérification des comptes. L'état des appointements de janvier $1807^{34}$ énumère quatre "employés à la comptabilité en matière du mobilier " : un préposé à la comptabilité en matière, un sous-inspecteur et deux commis expéditionnaires. Ces quatre personnes sont placées sous l'autorité du vérificateur, Sulleau, qui a derrière lui de nombreuses années d'expérience. Sur un total d'une petite trentaine d'employés, qu'il y en ait cinq qui soient affectés à la vérification des comptes est révélateur de l'importance accordée à cette opération.

31. Voir par exemple Arch. nat., $\mathrm{O}^{2} 524$, dossier 14, la lettre adressée par Desmazis au duc de Cadore, datée du 2 septembre 1812. Lui est joint le projet de budget de 1813.

32. Voir Arch. nat., $\mathrm{O}^{2} 556$, la lettre adressée par l'intendant général Daru à l'administrateur Desmazis, datée du 11 septembre 1807.

33. Voir Arch. nat., $\mathrm{O}^{2} 509$, dossier 9, l'allusion qui y est faite dans la lettre adressée par Desmazis à Daru, datée du 31 décembre 1810.

34. Voir Arch. nat., $\mathrm{O}^{2} 770, \mathrm{n}^{\circ} 46$. 
Une modification d'ampleur survient au niveau de l'étape suivante, qui est celle de la comptabilité. Nous avons vu que sous la Révolution le Garde-Meuble disposait d'un fonds d'avance, sur lequel il payait quelques menus frais de la catégorie des dépenses extraordinaires et urgentes. Un tel fonds n'existe plus sous l'Empire, où toutes les dépenses, sans exception ${ }^{35}$, sont réglées par la Trésorerie de la Couronne. Aucun membre du Mobilier impérial ne manie d'argent. L'administrateur se contente de demander le versement à la Trésorerie.

Le processus ne se résume pas là cependant. Car des acteurs extérieurs interviennent désormais dans le déroulement des opérations, alors que la Chambre des Comptes, responsable des biens de la Couronne, n'a pas réussi sous l'Ancien Régime à faire reconnaître son droit de contrôle sur les finances du Garde-Meuble ${ }^{36}$, et que, pendant la Révolution, les seules vérifications opérées à l'extérieur du GardeMeuble sont réalisées au sein du ministère de l'intérieur ou de la commission des Revenus nationaux. Dans un premier temps, la comptabilité du Mobilier impérial est examinée par le Comité de consultation des Bâtiments de la Couronne. Le contrôle des comptes du Garde-Meuble passe ensuite sous la responsabilité de trois auditeurs du Conseil d'État par le décret du 17 octobre $1807^{37}$. Dans les faits, ils ne sont jamais trois à être en poste en même temps, et les attributions géographiques prévues par l'article deuxième sont donc caduques. Canouville et Petiet sont nommés en 1808, et Petiet reste seul en fonction en $1809^{38}$. Puis Beyle et Lecoulteux de Canteleu ${ }^{39}$ prennent la relève pour les années 1810-1814. Pendant les Cent-Jours, l'auditeur du Garde-Meuble est Lacroix.

Le caractère radical de cette mesure de contrôle entraîne des lourdeurs administratives dont les fournisseurs sentent le poids. "Le Trésorier de notre Couronne ne paiera aucun compte du Garde-Meuble si la feuille qui constate la fourniture du meuble et le prix auquel il a été réglé au Garde-Meuble n'est certifiée par l'auditeur inspecteur ", affirme l'article troisième du décret du 17 octobre 1807. À cette décision répondent les plaintes des fabricants, telle celle formulée par Jacob-Desmalter le 27 décembre $1810^{40}:$ « Si vous n'aviez pas la bonté de nous faire expédier nos

35. Voir Mobilier national, "Enregistrement des lettres envoyées, an XIII-fin 1807 ", la lettre adressée par le Mobilier impérial à $\mathrm{M}$. le trésorier général de la Couronne, datée du 30 messidor an XIII (19 juillet 1805). Celui-ci est invité à faire mettre par son préposé à Bruxelles une somme de 3000 francs à la disposition du concierge du palais de Laeken, pour être consacrée au paiement de "diverses menues dépenses dont l'acquittement ne peut souffrir de retard." On a là typiquement une dépense qui, dix ans auparavant, aurait été réglée sur le fonds d'avance du Garde-Meuble.

36. Voir Pierre Verlet, Le Mobilier royal français. 2, Meubles de la Couronne conservés en France, avec une étude sur le Garde-Meuble de la Couronne, Paris, Picard, 1992, 220 p., p. 23-24. Le $1^{\text {er }}$ juin 1786 le roi tranche le long conflit en faveur du Garde-Meuble : les seuls inventaires à remettre à la Chambre des Comptes sont les inventaires des bijoux et diamants de la Couronne.

37. Voir Arch. nat., $\mathrm{O}^{2} 153$, l'extrait des minutes de la Secrétairerie d'État du palais de Fontainebleau.

38. Voir Arch. nat., $\mathrm{O}^{2} 782$, dossier 2.

39. Voir Arch. nat., $\mathrm{O}^{2}$ 556, dossier 1, la lettre adressée par Daru à Desmazis, datée du 27 août 1810, lui annonçant la nomination de Barthélémy Lecoulteux, fils du sénateur.

40. Voir Arch. nat., $\mathrm{O}^{2}$ 509, dossier 9, la lettre adressée par Jacob-Desmalter à Daru. 
mémoires et celui de M. Flamand pour la fin de l'année, vous nous mettriez dans un embarras insurmontable; il y a longtemps que les mémoires sont fournis, mais les formes administratives ont des longueurs qui écrasent un fabricant, celles qu'on vient d'ajouter entravent encore la marche; MM. les auditeurs, qui ont à faire une reconnaissance pure et simple des objets, la font attendre un temps infini."

\section{Des dépenses guidées par la conjoncture politique et économique}

La période de l'Empire se démarque de la décennie précédente par une explosion des dépenses dans le domaine du meuble, pour obvier à la crise et asseoir le régime notamment. Ces facteurs donnent une orientation particulière aux fournitures de mobilier.

Le Garde-Meuble du Premier Consul ne dispose pas encore de crédits pharaoniques. L'état des paiements restants à effectuer, "soit pour solde du mobilier des Tuileries et de Saint-Cloud, antérieurement à l'an XIII, soit pour travaux de décoration faits à la salle de spectacle du palais de Saint-Cloud, dans les années XI et XII, soit pour solde du service des camps et quartiers de Sa Majesté, à Boulogne, ou enfin pour fournitures faites lors du couronnement de Sa Majesté " ${ }^{41}$, daté du $1^{\text {er }}$ prairial an XIII (21 mai 1805), est révélateur. Le contenu de cet état pourrait indiquer simplement un dysfonctionnement au niveau de la rapidité de paiement des fournisseurs. Mais il semble plutôt qu'il révèle la médiocrité des sommes allouées au mobilier sous le Consulat.

Les choses commencent à changer avec la création du Mobilier impérial. Donnons quelques chiffres pour illustrer l'évolution des crédits accordés au mobilier ${ }^{42}$. En 1806, le total des sommes allouées au Garde-Meuble est de 1643486 francs; en 1807, il est de 1298500 francs; en 1808, il monte à 2019000 francs; en 1809, il est de 1480200 francs, avec 126435,53 francs de suppléments pour faire face aux dépenses excédant les crédits de l'année 1808 ; en 1810, il parvient à 2103000 francs; en 1811 enfin, dernière année pour laquelle on possède un budget et non pas seulement un projet de budget comme par la suite, il atteint 3229800 francs. Le mouvement global consiste en une stagnation autour de 1500000 francs dans les premières années, avec un pic à 2000000 de francs en 1808 , puis en une progression rapide avec le dépassement des 2000000 de francs en 1810 puis des 3000000 de francs en 1811 .

Pourtant, les années 1810-1811 marquent le début de la crise financière et industrielle qui survient dans les derniers temps de l'Empire. Des banques font faillite, des entreprises, trop endettées, sont aux abois, et les mauvaises récoltes de 1811 appauvrissent les campagnes. Ces éléments se répercutent sur le commerce. La crise économique, qui menace toujours de se transformer en crise sociale, vient s'ajouter aux difficultés religieuses qui secouent alors le pays. L'ensemble de ces questions constitue une menace véritable pour le régime napoléonien et incite

41. Voir Arch. nat., $\mathrm{O}^{2} 504$, dossier 4, le total de cet état est de 116277,51 francs.

42. Voir Arch. nat., $\mathrm{O}^{2} 562$, journal contenant les opérations du Mobilier impérial depuis les exercices an XIV (septembre-décembre 1805) et 1806. 
l'empereur à réagir. Alors que les gouvernements républicains avaient fait le choix, face à la crise, d'une politique exclusivement de rigueur qui avait étouffé le GardeMeuble, la réaction de l'empereur est différente : à la bonne gestion financière, il joint l'accroissement des crédits, afin de relancer l'économie de l'Empire.

"Rien ne pourrait plus contribuer à faire bénir par le peuple de Paris l'avènement de Sa Majesté Bonaparte au Trône impérial que l'établissement de ce GardeMeuble central qui répandrait des travaux et l'argent parmi toutes les classes industrieuses, et dont les ramifications feraient les mêmes effets dans les fabriques de Rouen, d'Amiens, de Lille et de Lyon ${ }^{43}$ ", écrit l'ancien inspecteur Bayard à l'intendant général de la Maison de l'Empereur le 21 messidor an XII (10 juillet 1804). Ce point de vue exclusivement économique adopté au sujet du Garde-Meuble, réducteur bien sûr, mais pragmatique, semble assez proche du regard que la période impériale a porté sur le Mobilier. Napoléon a pu avoir du goût pour le mobilier, cependant ce n'est pas l'approche esthétique d'un collectionneur ou amateur d'art qui préside au fonctionnement du Garde-Meuble, mais celle d'un dirigeant politique à la tête d'une nation en pleine évolution. Au cours des onze années que dure l'Empire, deux crises économiques d'ampleur nécessitent l'intervention de l'État pour relancer l'industrie.

La première a lieu en 1807 , en lien avec le blocus continental instauré par le décret de Berlin du 21 novembre 1806. A cette occasion, des études sont menées sur les "genres et espèces d'industries en souffrance " ${ }^{44}$, tandis qu'en parallèle l'intendant général centralise les demandes de secours formulées par les établissements touchés ${ }^{45}$. Un décret impérial met à la disposition de Daru une somme de 1600000 francs pour les commandes à faire pendant l'année ${ }^{46}$. Deux autres décrets, datés du 27 mars et du 11 mai 1807, octroient un prêt sur consignation à vingt-cinq manufacturiers, dont Feuchère, ${ }^{\circ}$ Thomire, et Jacob-Desmalter ${ }^{47}$. La maison Jacob-Desmalter reçoit 54225 francs pour occuper ses ouvriers qui, au nombre de trois cents à trois cent cinquante en temps ordinaire, sont passés à cent avec la crise. La plupart des débiteurs ne réussissent pas à se libérer totalement de leurs dettes et, en 1811 et 1812 , des marchandises sont confisquées parmi les objets déposés en consignation. Entre ainsi par exemple au Garde-Meuble le secrétaire à

43. Voir Arch. nat., $\mathrm{O}^{2}$ 556, dossier 1.

44. Voir Arch. nat., $\mathrm{O}^{2} 622$, dossier 1, un rapport sans date. Parmi les "industries en souffrance ", on peut citer par exemple les entreprises de gazes, crêpes, et passementerie, à Lyon et à Paris, qui " souffrent par le défaut de débouchés en Espagne et dans le Levant ".

45. Voir Arch. nat., $\mathrm{O}^{2} 622$, dossier 1 , un rapport sans date. Parmi les demandes adressées au gouvernement, citons celle de Castel, à la tête d'une filature de coton à Vincennes, qui demande 30000 francs pour prévenir la perte de son établissement.

46. Voir Arch. nat., $\mathrm{O}^{2} 622$, dossier 1, la lettre adressée par l'intendant au ministre de l'intérieur, datée du 30 avril 1807. Il lui adresse l'ampliation du décret impérial et le prévient qu'il a invité Desmazis à faire ses commandes auprès des manufactures qui, par leur état de souffrance, méritent le plus d'être encouragées.

47. Voir Jean-Pierre Planchon, Pierre-Benoît Marcion, 1769-1840, Saint-Rémy-en-l'Eau, M. Hayot, 2007, 239 p. 37. 
abattant en racine d'if des Indes, encadré de deux termes de bronze doré, l'intérieur en bois de citronnier, livré par Jacob-Desmalter pour 8000 francs en mars $1811^{48}$. L'empereur, par l'intermédiaire de son intendant et de son Garde-Meuble, passe des commandes et accorde des prêts, afin de pallier la réduction des débouchés causée par le blocus continental.

La seconde crise, qui dépasse celle de 1807 par son ampleur, se déclenche en 1810-1811. Par un décret du 10 mai 1811, l'empereur augmente de 350000 francs les fonds affectés par le budget de cette année aux dépenses de mobilier et ordonne l'emploi de cette somme en ouvrages d'ébénisterie et de menuiserie. L'objet de ce décret est de donner de l'activité aux ouvriers parisiens de cette classe qui, dans ce moment, manquent de travail. Napoléon souhaite que les ouvrages que l'on commande pour cette somme de 350000 francs occupent de 1600 à 2000 ouvriers pendant un mois et demi au moins, ce qui est réalisable puisque cette somme, distribuée en quarante-cinq jours, donne près de 8000 francs par jour et, en supposant que la matière travaillée absorbe la moitié du prix, il reste encore le salaire de 2000 ouvriers à 2 francs par jour chacun. Il est nécessaire de veiller surtout à ce que ce fonds ne soit pas employé pour payer des meubles déjà existants dans les magasins des marchands de Paris ${ }^{49}$. Pour répartir les commandes, Desmazis s'appuie ensuite sur les listes de fabricants de meubles parisiens qui lui sont fournies par le préfet de Police de Paris ${ }^{50}$. Certains d'entre eux, sans doute peu habitués à ce type de commandes et aux exigences qui leur sont assorties, ne peuvent honorer leurs engagements ${ }^{51}$.

Mais cette somme de 350000 francs destinée à venir en aide aux fabricants parisiens est bien peu de choses en comparaison des moyens mis en œuvre à Lyon, dès décembre 1810. Dans une lettre du 20 décembre $1810^{52}$, le grand maréchal du Palais explique à Desmazis que Sa Majesté veut venir en aide aux fabriques de Lyon qui sont en souffrance. Pour cela, il prévoit des commandes pour deux millions de francs payables dans les trois ou quatre premiers mois de 1811, et même en deux mois, si cela est nécessaire, pour mettre en activité une grande quantité de métiers sans occupation. Desmazis est invité à prendre contact avec le ministre de l'intérieur, puis à réunir les négociants de Lyon avec les employés du GardeMeuble pour s'entendre sur les commandes, en grande partie destinées à Versailles.

48. Voir Sylvain Laveissière (sous la dir. de), Napoléon et le Louvre, Paris, Fayard, 2004, 255 p, p. 167-168.

49. Voir Arch. nat., $\mathrm{O}^{2} 537$, dossier 4, la lettre adressée par l'intendant général Pierre Daru à Desmazis, datée du 10 mai 1811. Il lui envoie le décret de Sa Majesté assorti de recommandations pratiques.

50. Voir Arch. nat., $\mathrm{O}^{2} 537$, dossier 4, les rapports adressés par les services de police de Paris à Desmazis, datés des 7 et 9 mai 1811 .

51. Voir Arch. nat., $\mathrm{O}^{2}$ 537, dossier 4, la lettre adressée par le duc de Cadore, intendant général, à Desmazis, datée du 29 février 1812. Il y évoque notamment un certain sieur Thomas Michel, qui s'était engagé à livrer au Garde-Meuble quatre secrétaires et quatre commodes en bois français montant à 880 francs, mais ne peut remplir ses engagements. Il approuve le fait d'annuler sa soumission et de charger de cette fourniture le sieur Lemaître, ébéniste, qui a soumissionné les mêmes meubles, aux mêmes prix et conditions que le sieur Michel.

52. Voir Arch. nat., $\mathrm{O}^{2}$ 521, dossier 1. 
Le but doit être bien rempli, mais les intérêts de l'empereur ne doivent pas s'en trouver lésés pour autant. Si l'on pouvait faire autant d'effets avec moins d'argent, ce serait préférable. Le Mobilier impérial n'est pas le seul à gérer ces commandes, car Desmazis demande la constitution d'un syndicat, sous l'autorité du préfet, pour servir d'intermédiaire entre les fabriques lyonnaises et le Garde-Meuble, et surveiller leurs travaux ${ }^{53}$.

En 1807 et 1810-1811, les commanditaires se faisant rares en raison des crises, l'État se substitue à eux par l'intermédiaire de l'intendant général de la Maison de l'Empereur et du Mobilier impérial. Donner du travail aux entrepreneurs et aux manufactures est capital pour relancer l'économie, conserver la paix sociale et préserver la stabilité politique.

La période de l'Empire est donc pour le Garde-Meuble un moment de rationalisation de ses dépenses. Elles font l'objet d'un budget, qui est préparé plusieurs mois à l'avance et rectifié si besoin est. C'est également un moment de rigueur dans la gestion de l'institution. Les contrôles sont multiples tant à l'intérieur qu'à l'extérieur de l'institution. On remarque d'ailleurs qu'il n'y a pas de trace de vols ou de dégradations volontaires. Cette rigueur s'accompagne d'une logique de relance, qui profite au Mobilier impérial, dont les crédits sont multipliés, mais subordonnés aux objectifs économiques et politiques du régime.

Entre finances et arts, la priorité semble aller aux arts sous l'Ancien Régime, période où le roi et ses proches ne rechignent pas à faire des dépenses somptueuses pour se meubler et décorer leurs intérieurs. L'état d'esprit se modifie en profondeur à partir des années révolutionnaires. Le Garde-Meuble, institution liée depuis l'origine aux fastes de la monarchie, se voit imposer une rigueur budgétaire inconnue jusqu'alors et se transforme même en institution productrice, avant de se voir supprimer par manque d'intérêt pour ses activités jugées encore trop coûteuses. Tiré du néant sous l'Empire et doté de crédits considérables, en augmentation durant l'ensemble de la période, le Garde-Meuble voit sa gestion contrôlée avec une rigueur qui nuit à l'occasion à ses fournisseurs, et ses fonds orientés en fonction de la conjoncture économique du moment. Entre finances et arts, ce sont désormais les finances qui tiennent la première place. Le soutien aux arts désintéressé, ou motivé seulement par des considérations de prestige, disparaît.

Aleth TISSEAU DES ESCOTAIS conservateur des bibliothèques stagiaire à l'ENSSIB

53. Voir Arch. nat., $\mathrm{O}^{2} 521$, dossier 1, la lettre adressée par Desmazis à Daru, datée du 27 décembre 1810. 